\title{
Synthetic Cannabinoid Use in a Psychiatric Patient Population: A Pilot Study
}

\author{
Stella Welter ${ }^{a, b}$ Caroline Lücke Alexandra Philomena Lam $^{\mathrm{a}, \mathrm{b}}$ Christina Custal ${ }^{\mathrm{c}}$ \\ Sebastian Moeller ${ }^{a}$ Peter Sörös $^{b} \quad$ Christiane M. Thiel $^{\mathrm{a}} \quad$ Alexandra Philipsen $^{\mathrm{b}}$
}

Helge H.O. Müller ${ }^{b, c}$

${ }^{a}$ Department of Biological Psychology, and ${ }^{b}$ Department of Psychiatry and Psychotherapy, Carl von Ossietzky

University of Oldenburg, Faculty of Medicine and Health Sciences, Karl-Jaspers-Klinik, Bad Zwischenahn, and

'Department of Psychiatry and Psychotherapy, Friedrich-Alexander University of Erlangen-Nuremberg,

Erlangen, Germany

\section{Keywords}

Cannabinoid consumption · Synthetic cannabinoid

consumption · Psychosis vere in SC and NC users, respectively. Conclusions: NC and SCs may cause different symptom clusters. These relationships should be further evaluated.

(c) 2017 S. Karger AG, Base

\begin{abstract}
Background: Consumption of natural cannabis (NC) and synthetic cannabinoids ( $\mathrm{SCs}$ ) has been associated with psychotic disorders. We compared the prevalence of use, consumer profiles, and psychosis-inducing potential of NC and SCs in a specific high-risk population. Methods: This prospective pilot study included 332 patients (18-64 years, mean 36.83, SD 13.33). Patients' sociodemographics and medical histories as well as illicit substance use and psychiatric symptom histories were collected using a drug consumption survey that assessed the use of new psychoactive substances and the Psychotic Symptoms Interview. Results: In total, $7.2 \%$ of all patients, $10.6 \%$ of psychotic patients, and $4.5 \%$ of nonpsychotic patients reported SC consumption. Compared with SCs, NC was consumed much more frequently by its users (mean 222.73, SD 498.27). NC and SC use induced persistent psychosis. Psychotic symptoms were first experienced by patients with a history of NC or SC use during intoxication and persisted after cessation ( $>1$ year) of drug use. Positive and negative symptoms tended to be more se-
\end{abstract}

(c) 2017 S. Karger AG, Basel

E-Mail karger@karger.com www.karger.com/ear

\section{Background}

Synthetic cannabinoids (SCs, or Spice) were initially developed in the 1960s for use as pain-relieving agents. However, SC compounds that exerted the desired therapeutic effects without simultaneously inducing psychoactive side effects could not be developed. Since the mid2000s, the international drug market has been flooded with new designer drugs, including Spice, which is the most commonly used SC [1-3]. These products have effects similar to those of natural cannabis (NC), are deceptively marketed as 'natural' herbal incense, and are widely sold on the Internet without age restrictions. Common street names of these drugs include Spice (the most common), Aroma, K2, Fake Weed, Mojo, and Dr. Feel Good

This study is registered under 082/2016 (CvO Oldenburg). A.P. and H.H.O.M. contributed equally to this work. 
[4-7]. Increasing evidence indicates that SCs have potentially dangerous side effects $[4,8-11]$. However, to circumvent drug legislation, manufacturers of these substances continue to develop new compounds with slightly modified chemical structures. These modifications enable manufacturers to legally distribute the substances until they are registered and banned by law [12-15].

Because these drugs are widely and legally available, they are also very popular. SC consumption seems to be particularly common among young adults. A 2014 survey of 15-18-year-old German adolescents showed that 6\% had consumed an herbal smoke blend containing SCs at least once in their life and that $1 \%$ had consumed such a blend in the past 30 days (Bernard, Kamphausen, Egger, Sarvari, \& Müller, 2015). An estimated 1-10\% of the general population has consumed SCs; however, data regarding the prevalence of SC use are sparse.

SCs can be developed rapidly and exist in several different forms. Additionally, they have strong cannabimimetic effects, can induce euphoria and relaxation, and enable patients to feel calm and safe $[10,11,16,17]$. However, accumulating evidence indicates that SCs can induce serious side effects, including psychosis $[9,18-20]$. The association between $\mathrm{NC}$ use and the risk of psychosis is controversial; however, less is known about the potential of SCs to cause psychosis. SCs are believed to have a greater potential to induce psychosis than NC because they have higher affinity for cannabinoid receptor type 1 (CB1) $[18,19,21,22]$. Moreover, previous studies suggest that the side effects induced by SCs may be more severe and may occur more frequently than those induced by $\mathrm{NC}$ [22-30].

Previous studies have also shown that psychotic patients consume more cannabis and are more likely to experience adverse effects from cannabis use than non-psychotic patients (Green et al. [31]) [22]. In addition, patients with psychotic disorders (van Os et al., 2002 [56]), as well as patients who are genetically predisposed to psychotic disorders, may be more vulnerable to cannabinoid-induced psychotic reactions than patients without psychotic disorders [32-35]. Previous studies have reported cases in which patients' initial SC-induced psychotic symptoms persisted well beyond their period of intoxication [9, 11, 34-37].

Most of the existing knowledge regarding cannabisinduced psychosis comes from case reports, poison control center data, and online surveys [37-40]. Laboratory studies on the effects of SCs in humans have reported on the acute psychosis-inducing actions of SCs, but few studies have explored the effects of these compounds in a large clinical sample [41, 42]. Given the high rate of cannabis consumption among psychotic patients and these patients' increased vulnerability to the adverse effects of cannabis use, the scarcity of systematic data on the prevalence of SC use in this population is surprising.

Therefore, this study aimed to investigate the prevalence of SC use, the consumer profiles of SC users, and the psychosis-inducing potential of SCs in a specific psychiatric patient population. Psychotic patients were included in this study to enable further insight into how SCs influence the course of psychotic disorders.

\section{Objectives}

We formulated and addressed 4 main research questions to gain a better understanding of the prevalence of SC use, the consumer profiles of SC users, the psychosisinducing effects exerted by SCs and $\mathrm{NC}$, and the relationship between these effects and psychotic illnesses:

- How frequently are SCs consumed in general psychiatric patient populations and, in particular, among psychotic patients?

- Which demographic variables are associated with SC use?

- How frequently do psychotic reactions to either drug type occur; how severe are these reactions; what symptoms do the affected patients experience during these reactions; are the reactions acute or prolonged; and do the drugs exacerbate preexisting psychiatric symptoms in psychotic patients?

- Are psychotic patients more likely than non-psychotic patients to experience symptoms of psychosis when using either type of drug?

We also developed a series of hypotheses regarding the main research objectives. First, we speculated that the prevalence of SC use would be higher in psychiatric patients than in the general population and that the prevalence of SC use would be highest among psychotic patients. Second, we surmised that the risk factors for SC use would be young age, male gender, psychotic illness, smoking, and (other) drug use. Third, we hypothesized that SCs elicit more frequent and more severe psychotic reactions than NC. Finally, we suspected that compared to non-psychotic patients, psychotic patients would experience more frequent and more severe symptoms of acute psychosis due to SC or NC use.

\section{Methods}

Participants

A total of 332 individuals who were undergoing treatment at the inpatient clinic of the Institute of Psychiatry at the University 
of Oldenburg participated in this study. Patients from the geriatric psychiatric unit ( $>65$ years) were excluded from the study, as drug use was expected to be uncommon among patients in this age group. However, all other patients, regardless of diagnosis, were included in the study to prevent selection bias from affecting the results and to assess patients in younger age groups, who are at higher risk for using psychotropic substances, including cannabinoids and other substances. The participants were 18-64 years (mean 36.83, SD 13.33). Data were collected from March-December 2016. Of the patients included in the full cohort, $48 \%$ were from a closed ward and $45.5 \%$ were psychotic upon admission. The most common diagnoses affecting these patients were major depressive episodes without psychotic features $(27.1 \%)$, paranoid schizophrenia $(21.1 \%)$, borderline personality disorder (13.9\%), and schizoaffective disorder (11.7\%). Data regarding all diagnoses present in the sample and the frequencies of these diagnoses are presented in Table 1 . To acquire epidemiological data, we administered a preselection survey that asked psychotic and non-psychotic patients from the psychiatric clinic about their drug consumption behavior (focusing specifically on their history of NC and SC consumption) and their demographic characteristics. All patients also underwent routine screening for cocaine, heroin, and amphetamine use during treatment. Patients identified as SC or NC users were asked to participate in an interview about the psychotic effects of the drugs. The interviews retrospectively acquired information about the occurrence of specific psychotic symptoms at the following 3 time points: before using the drug for the first time, during drug use (72-h time period), and after cessation of drug use. All patients who completed the above assessments had been inpatients at the clinic for at least 5 days; therefore, we assumed that they had not used drugs for a minimum of 5 days. Moreover, we also obtained data on the frequencies with which patients experienced specific symptoms during the abovementioned time points. We did not interview a matched non-psychiatric population in this study. All participants provided written consent to participate in the study, which was approved by the Ethics Committee of the Faculty of Medicine/Carl-von Ossietzky University Oldenburg.

Of the 332 patients interviewed during the first part of the study, 23 who had used SCs and 26 who had used NC participated in the second part of the study; this second stage included an interview that gathered information on the associations between patients' psychotic symptoms and drug use. All 7 of the patients who had alternated between using SCs and NC and all 4 of the patients who had concomitantly used SCs and NC reported using both substances in the past ( $>1$ year before interviewing); additionally, all SC users reported using NC in the past. The NC users included in this portion of the study were age- and gender-matched with SC users. The 2 groups had similar proportions of patients with a history of psychotic symptoms. Two patients in the SC group had limited recollections of their drug use experiences and were thus excluded from the analysis. Therefore, the final SC-user sample comprised 21 patients. There was overlap between the 2 groups, as the majority of SC users had used NC previously (more than once within the year prior to the study). Figure 1 shows a flow diagram containing information about the patients who were initially approached about study participation and the patients who participated in each step of the data collection process. The information collected during the interviews is summarized in Table 2, and the data regarding the distribution of diagnoses among the patient samples are included in Tables 3 and 4.

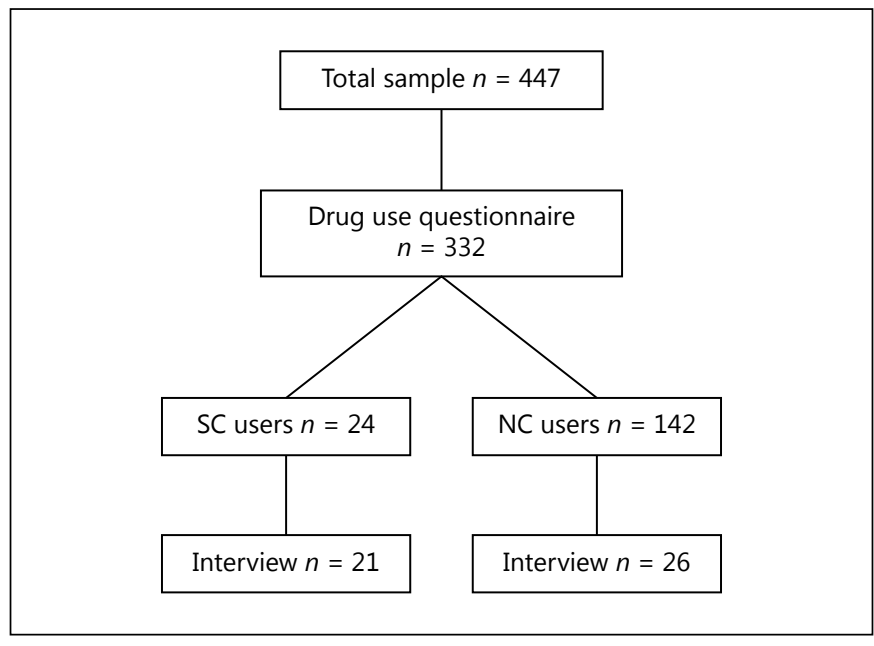

Fig. 1. Flow diagram of the participants selection process.

Table 1. Diagnoses of the patients in the total sample

\begin{tabular}{ll}
\hline ICD10 diagnosis & Proportion, $n$ \\
& $\%$
\end{tabular}

Major depressive disorder without

psychotic features

Paranoid schizophrenia

$\begin{array}{rr}27.1 & 90 \\ 21.1 & 70 \\ 14.5 & 48 \\ 11.7 & 39 \\ 7.8 & 26 \\ 4.8 & 16 \\ 3.9 & 13 \\ & \\ 2.4 & 8 \\ 1.5 & 5 \\ 1.5 & 5 \\ 0.9 & 3 \\ 0.6 & 2 \\ 0.6 & 2 \\ 0.6 & 2 \\ 0.6 & 2 \\ 0.3 & 1 \\ 100 & 332\end{array}$

Borderline personality disorder

Schizoaffective disorder

Bipolar disorder

Schizophreniform disorder

Organic affective disorder

Major depressive disorder with psychotic

features

Adjustment disorder

Somatoform disorder

Manic episode without psychotic symptoms

Anorexia nervosa

Generalized anxiety disorder

Cannabis dependence

Unknown diagnosis

Disorganized schizophrenia

Total

0 0 39 26 13 5 332

\section{Drug Consumption Survey}

The drug consumption survey included questions regarding the consumption of new psychoactive substances (NPS), including SCs, and other drugs, as well as questions designed to collect sociodemographic data. The cover sheet included information about the drugs that were considered NPS and their most common brand names. The participants were then asked to indicate whether they had ever used NPS and, if so, what type of NPS that they had used and how they had obtained the substance. Furthermore, the patients were asked to provide information regarding the frequency 
Table 2. Demographic characteristics of the patients in the SC and NC user samples

\begin{tabular}{lll}
\hline & SCs & NC \\
\hline $\begin{array}{ll}\text { Age, years } \\
\quad \text { Mean (SD) }\end{array}$ & & \\
$\quad$ Range & $25.6(5.8)$ & $31.8(10.5)$ \\
Gender, \% $(n)$ & $19-36$ & $19-56$ \\
$\quad$ Male & & \\
$\quad$ Female & $71.4(15)$ & $61.5(16)$ \\
Closed ward, \% $(n)$ & $28.6(6)$ & $38.5(10)$ \\
$\quad$ Yes & & \\
$\quad$ No & $81(17)$ & $42.3(11)$ \\
Psychotic on admission, \% $(n)$ & $19(4)$ & $57.7(15)$ \\
$\quad$ Yes & $71.4(15)$ & $61.5(16)$ \\
$\quad$ No & $28.6(6)$ & $38.5(10)$ \\
\hline
\end{tabular}

Table 3. Diagnoses of the patients in the SC user sample

\begin{tabular}{llr}
\hline ICD10 diagnosis & Proportion, \% & $n$ \\
\hline Paranoid schizophrenia & 47.6 & 10 \\
Depression without psychosis & 19 & 4 \\
Schizoaffective disorder & 14.3 & 3 \\
Schizophreniform disorder & 4.8 & 1 \\
Anorexia nervosa & 4.8 & 1 \\
Disorganized schizophrenia & 4.8 & 1 \\
Mania without psychosis & 4.8 & 1 \\
Total & 100 & 21 \\
\hline
\end{tabular}

Table 4. Diagnoses of patients in the NC user sample

\begin{tabular}{lcr}
\hline ICD10 diagnosis & Proportion, \% & $n$ \\
\hline Paranoid schizophrenia & 46.2 & 12 \\
Borderline & 11.5 & 3 \\
Depression with psychosis & 7.7 & 2 \\
Bipolar disorder & 7.7 & 2 \\
Adjuvant disorder & 3.8 & 1 \\
Cannabis dependence & 3.8 & 1 \\
Disorganized schizophrenia & 3.8 & 1 \\
Depression without psychosis & 3.8 & 1 \\
Schizoaffective disorder & 3.8 & 1 \\
Organic affective disorder & 3.8 & 1 \\
Mania without psychosis & 3.8 & 1 \\
Total & 100 & 26 \\
\hline
\end{tabular}

and duration of their drug use, as well as the age at which they had first used the drug. The participants were also asked to indicate whether they had ever consumed any other drugs (e.g., cocaine, heroin, amphetamines, cannabis, or others). Finally, patient's age, gender, education, and personal and familial psychiatric history were assessed. Data regarding the patients' current diagnoses and whether they presented with psychosis on admission were retrieved from their medical records.

\section{Psychotic Symptoms Interview}

A modified version of the Positive and Negative Syndrome Scale [43] was used to assess the relationship between cannabinoid consumption and psychotic symptoms. The Positive and Negative Syndrome Scale is a diagnostic instrument that is used to evaluate symptom severity in patients with schizophrenia. Each patient undergoes a semi-structured clinical interview in which he or she is asked questions regarding 30 symptoms; the responses are rated on a Likert scale ranging from 1 to 7 to determine the presence of symptoms and their severity. This study assessed the following 9 symptoms, which have been shown to be commonly associated with cannabinoid use [24], to determine their occurrence and severity in the patient sample: 5 positive symptoms, that is, delusions, conceptual disorganization, hallucinations, grandiosity, and delusions of persecution, and 4 negative symptoms, that is, blunted affect, emotional withdrawal, poor rapport, and motor retardation. The patients were first prompted to recall situations in which they had consumed only NC or SCs and were then asked to describe the circumstances surrounding their exposure(s) to each drug. This procedure was used to enhance memory retrieval and to simultaneously evaluate the clarity of the patients' memories. Patients with limited memories regarding the above events were excluded from the study. The interviewer explained each of the above symptoms to the patients individually and in layman's terms. Patients were then asked to indicate whether they had ever experienced the symptom during SC/NC consumption (with a 24 -h time period), to quantify the average severity of their symptoms (i.e., across drug exposures $)$ on a scale from 1 to $7(1=$ absent, $2=$ minimal, $3=$ mild, $4=$ moderate, $5=$ moderately severe, $6=$ severe, $7=$ extreme) and, if possible, to describe the experience to the interviewer. Furthermore, patients were asked whether they had ever experienced the symptom prior to their first use of SCs or NC and whether they had ever experienced the symptom after cessation ( $>4$ weeks) of drug use (standard drug screens were routinely performed for all inpatients to verify the accuracy of their responses). Patients were also asked to describe the severity of the symptom over time and to rate the severity of the symptom before first drug use, during drug use, and after cessation according to the abovementioned 1-7 scale.

\section{Statistical Analysis}

Consumer Profile

To investigate the associations between SC use and demographic variables, we performed logistic regression analysis (using backward stepwise elimination based on the Wald statistic) to determine whether age, gender, smoking status, frequency of cannabis use, history of drug use (other than cannabis), and education were predictors of SC use.

Comparison of Acute Psychotic Reactions between the

Drug Groups

Logistic regression analysis and Mann-Whitney U tests were applied to determine whether acute psychotic reactions occurred more frequently or were more severe in patients who used a particular drug. For this assessment, drug type was considered an independent variable (IV), and the binary outcome 'acute psychotic reaction' was included as a dependent variable (DV). This analysis was performed 
for the symptom clusters associated with each type of reaction, that is, an "acute positive psychotic reaction" and an "acute negative psychotic reaction" (each coded as a binary "yes/no" response). These symptom clusters were used as DVs in separate analyses.

Differences in the severity of the acute symptoms elicited by the drugs were tested using independent samples Mann-Whitney $U$ tests. These analyses were not corrected for multiple tests because of the exploratory nature of the study. Drug type was included as an IV, and the severity scores of each symptom elicited by drug use were considered DVs. Moreover, we calculated summary scores for the severity of each patient's positive and negative symptoms and an overall score for the severity of acute symptoms, and these parameters were compared between users of each drug according to the tests described above.

\section{Comparison of Symptom Trajectories between the}

Drug Groups

To determine whether SCs and NC could exacerbate preexisting psychotic symptoms, we conducted a Friedman test to assess the severity of the patients' symptoms over time. This test is a nonparametric alternative to one-way repeated measures ANOVA and was used to investigate the changes in symptom scores over 3 or more time points.

Influence of Psychotic Illness on Psychotic Reactions

The relationship between psychotic illness on admission and the occurrence of acute psychotic reactions following the use of $\mathrm{NC}$ or SCs was assessed using separate chi-square tests, which compared the incidences of psychotic reactions after the use of each drug between subgroups of psychotic and non-psychotic patients.

To determine the effect of psychotic illness on the severity of acute psychotic reactions to NC or SCs, we performed Mann-Whitney $U$ tests. For these assessments, psychotic illness was used as an IV, and the acute severity scores for each symptom, as well as the overall, positive and negative summary scores, were used as DVs.

Influence of Drug Use Frequency on Psychotic Reactions/

Statistical Tests

To determine whether the frequency of SC or NC use affected or whether an acute psychotic reaction was induced by either drug, we conducted logistic regression analyses in which the frequency of drug use was an IV and the occurrences of general, positive and negative symptoms (yes/no) were DVs. For the ANOVA, symptom severity was used as a DV.

Regressions of the percentage of exposures to each drug that resulted in a psychotic reaction (average across all symptoms) were conducted to determine whether SCs and NC differed in the prevalence of elicited psychotic reactions.

Data processing and analysis were performed using SPSS Statistics 24 (IBM, Armonk, NY, USA).

\section{Results}

\section{Prevalence of SC Use}

Of all patients enrolled in the study, $7.2 \%$ reported a history of SC consumption. Overall, $79.2 \%$ of patients enrolled in the study indicated that they did not intend to use SCs in the future, and $83.0 \%$ of SC users indicated that they did not intend to use SCs again.

SC use was 2 times higher among psychotic patients than among non-psychotic patients, and this difference was significant $\left(\chi^{2}[1]=4.56, p=0.033\right)$, namely, $10.6 \%$ of psychotic and $4.5 \%$ of non-psychotic patients reported a history of SC consumption. No patients reported using other psychotropic substances while using SCs or NC ( $>2$ years before the interview).

\section{Consumer Profile}

The average age of first SC use was 23.52 years (SD 5.29). Logistic regression analysis of the relationship between SC use and various demographic variables yielded a model in which gender, smoking, and frequency of cannabis consumption predicted SC use. Comparing the full model with a constant-only model led to significant results, indicating that the predictors could reliably distinguish between SC users and nonusers $\left(\chi^{2}[3]=38.488, p<\right.$ 0.001 , Nagelkerke's R squared $=0.274)$. The most significant determinants of SC use were smoking and frequency of cannabis use ( $p=0.001 /$ smoker; $p=0.003 /$ frequency). Gender ( $p=0.059)$ was only a marginally significant predictor of SC use. No other factors were found to predict SC use. The results of this analysis are shown in Table 5.

\section{Frequency of Drug Use among SC and NC Users}

The frequency of SC use among SC users ranged from once to 100 times (mean 9.88, SD 21.83). The majority of former NC-only users (62.5\%) had consumed SCs only once. However, the frequency with which NC was consumed by its users was significantly higher than the frequency with which SCs were consumed by their users. Specifically, NC users reported using NC a mean of 222.73 times (SD 498.27), and the frequency of NC use in these patients ranged from once to 4,000 times. As was the case for patients in the SC group, the majority of patients $(61.2 \%)$ in the NC group had used SC only once.

\section{Psychotic Reactions to SC/NC Use}

\section{Acute Psychotic Reactions}

SCs. Sixteen of 21 (76.2\%) SC users reported experiencing at least one of the symptoms listed in Figure 2 (e.g., persecution, delusion, hallucinations) while using SCs. The most common symptoms experienced by SC users were motor retardation and blunted affect (both symptoms affected $42.9 \%$ of users) and delusions of persecution and disorganization (both symptoms affected 23.8\% of users). All symptoms assessed were experienced by at least one of the participants. 
Fig. 2. Proportions of exposures associated with acute symptoms (across subjects). The error bars represent standard errors.

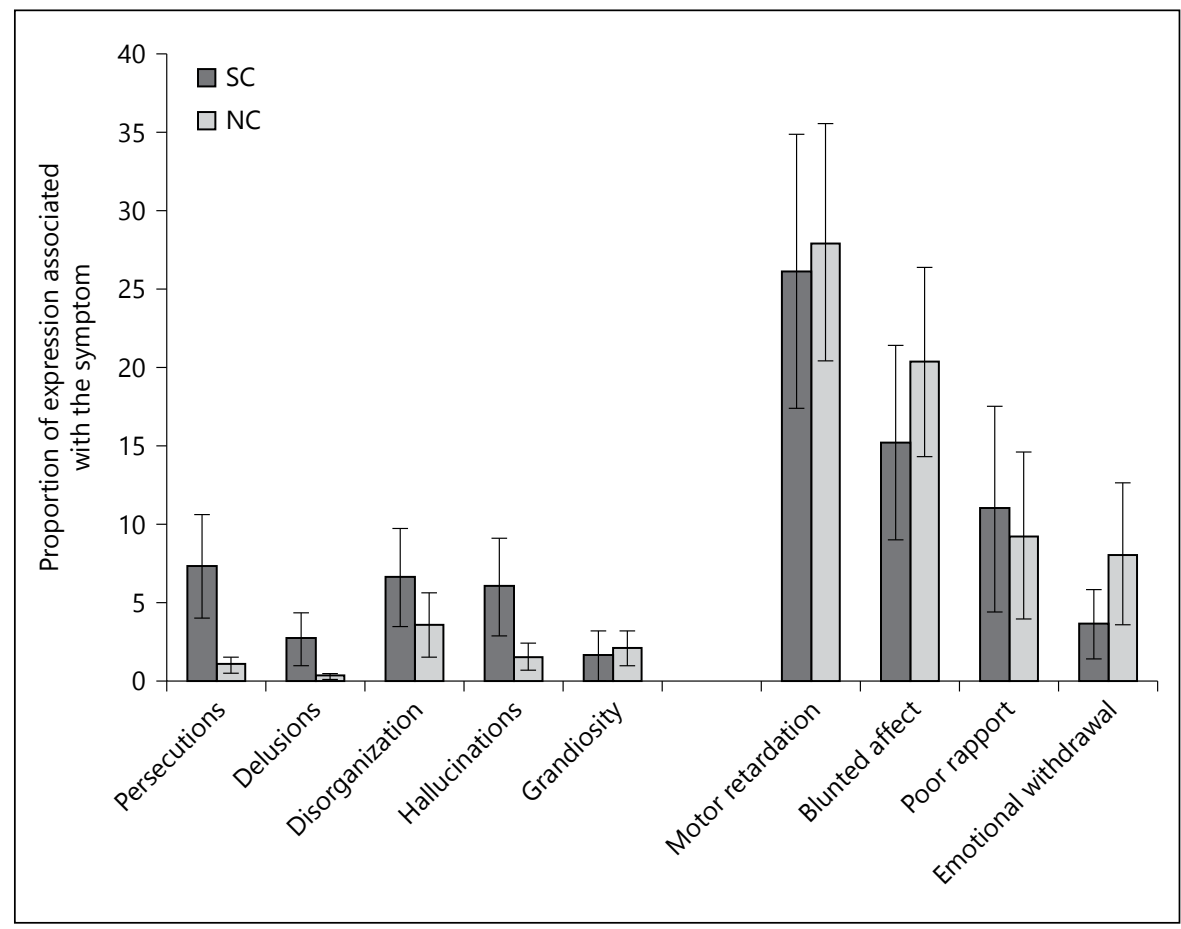

Table 5. Summary of hierarchical logistic regression analysis for variables predicting lifetime SC use. Smoking and frequency of cannabis use were significant predictors of SC use, and sex was a marginally significant predictor of SC use $(p=0.059)$

\begin{tabular}{|c|c|c|c|c|c|c|c|c|}
\hline \multirow[t]{2}{*}{ Model } & \multirow[t]{2}{*}{ B } & \multirow[t]{2}{*}{$\mathrm{SE}$} & \multirow[t]{2}{*}{ Wald } & \multirow[t]{2}{*}{ d.f. } & \multirow[t]{2}{*}{ Significance } & \multirow[t]{2}{*}{$\operatorname{Exp}(B)$} & \multicolumn{2}{|c|}{$95 \%$ CI for $\operatorname{Exp}(B)$} \\
\hline & & & & & & & lower & upper \\
\hline Gender & -0.976 & 0.518 & 3.552 & 1 & 0.059 & 0.377 & 0.137 & 1.040 \\
\hline Smoker & 2.547 & 0.755 & 11.374 & 1 & 0.001 & 12.772 & 2.906 & 56.125 \\
\hline Frequency cannabis use & 0.002 & 0.001 & 9.035 & 1 & 0.003 & 1.002 & 1.001 & 1.003 \\
\hline Constant & -4.296 & 0.728 & 34.434 & 1 & 0.000 & 0.014 & & \\
\hline
\end{tabular}

NC. Twenty-one of $26(80.8 \%)$ NC users reported experiencing at least one psychotic symptom while using NC. The most common symptoms experienced by NC users were blunted affect (50\% of users), motor retardation ( $46.2 \%$ of users), disorganization ( $23.1 \%$ of users), and emotional withdrawal (19.2\% of users; Fig. 2). As was the case in the SC group, all symptoms assessed in the NC group were experienced by at least one of the participants.

\section{Persistent Psychotic Reactions}

SCs. Two male patients (aged 24 and 29 years) reported experiencing worsening psychotic symptoms (delusions of persecution and hallucinations for one and per- sistent delusions and hallucinations for the other) after first consuming SCs.

NC. Two non-psychotic patients (a 20 -year-old female and 19-year-old male) reported experiencing blunted affect for the first time while using cannabis and indicated that this symptom persisted after cessation of cannabis use.

\section{Exacerbations of Preexisting Symptoms}

None of the patients experienced exacerbations of preexisting symptoms, that is, symptoms that occurred during drug use and became more severe after cessation of drug use. 
Comparisons of the Frequencies of Psychotic Reactions to NC and SCs

Acute Psychotic Reactions

Prevalence. Logistic regression analyses were performed to compare the prevalence rates of acute psychotic symptoms and positive or negative symptoms between SC and NC users. These analyses showed that the prevalence of psychotic symptoms and positive or negative symptoms did not differ between the 2 groups, suggesting that the prevalence of acute psychotic reactions did not differ between SC and NC users.

Given the different frequencies with which NC and SC users were exposed to their respective drugs, we analyzed the relationship between the incidence of acute psychotic symptoms and the frequency of drug use for each patient. The mean percentage of each symptom associated with all exposures assessed and the corresponding standard deviations are shown in Figure 2, which also presents data regarding the percentages of drug exposures that were associated with each symptom experienced by the subjects.

Regression analysis of the percentages of specific drug exposures resulting in a psychotic reaction (averaged across all symptoms) did not yield significant results, indicating that there was no difference between SCs and NC with regard to the prevalence of psychotic reactions occurring during their use. Separate multi-regression analyses assessing the prevalence of positive and negative symptoms associated with drug use showed that positive symptoms $(F[1,45]=3.69, p=0.061)$ but not negative symptoms were marginally and significantly associated with drug use. However, the data shown in Figure 2 indicate that positive symptoms (with the exception of grandiosity) were more frequently associated with SC use and that negative symptoms (with the exception of poor rapport) tended to be more frequently associated with $\mathrm{NC}$ use. Moreover, both NC and SC users experienced negative symptoms (right side; Fig. 2) much more frequently than positive symptoms.

Severity. Only patients who experienced the listed symptoms (severity rating over 1 year $=$ absent, $p=0.001$ ) were included in this analysis.

Tests comparing the severity of the acute symptoms elicited by the 2 drugs revealed significant differences between groups in the summary score for negative symptoms $(\mathrm{U}=170.50, p=0.026)$. Specifically, NC users experienced significantly more severe negative symptoms than SC users. Figure 3 shows a side-by-side boxplot depicting the severity of the negative symptoms experienced by users of the 2 drugs. There were no significant differences in

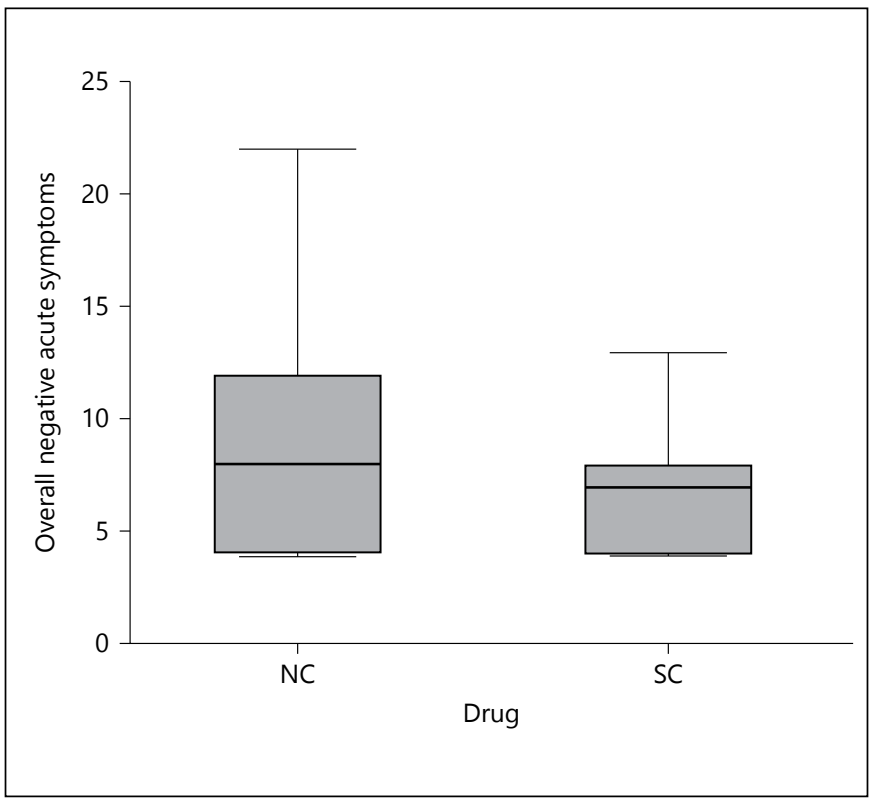

Fig. 3. Comparison of the severity of negative symptoms between the different drug types.

the severity of individual positive symptoms or in the overall severity of positive symptoms between the 2 groups.

The mean severity scores indicated that positive symptoms (except grandiosity) tended to be more severe in SC users and that negative symptoms tended to be more severe in NC users. Poor rapport was equally severe between the 2 groups. Friedman tests were conducted to assess the changes in the severity of patients' symptoms over time, that is, the changes in their severity scores over the following 3 time points: before, during, and after drug use. The severity of disorganization and poor rapport changed significantly in SC users, and the severity of blunted affect and motor retardation changed significantly in both SC and NC users. These results indicated that there were significant differences in the severities of the above symptoms between the predrug use, current drug use, and post-drug use time points. To determine whether the patients' symptoms had worsened from before to after drug use, we conducted post hoc tests (Wilcoxon's signed rank tests) to identify the time points between which the severities of the symptoms differed (Figure 4 gives a comparison of the severity between the examined substances). We noted no differences in the severity of the symptoms between pre- and post-drug use, indicating that the individual symptoms were not exacerbated after drug consumption. However, the severity of the patients' 


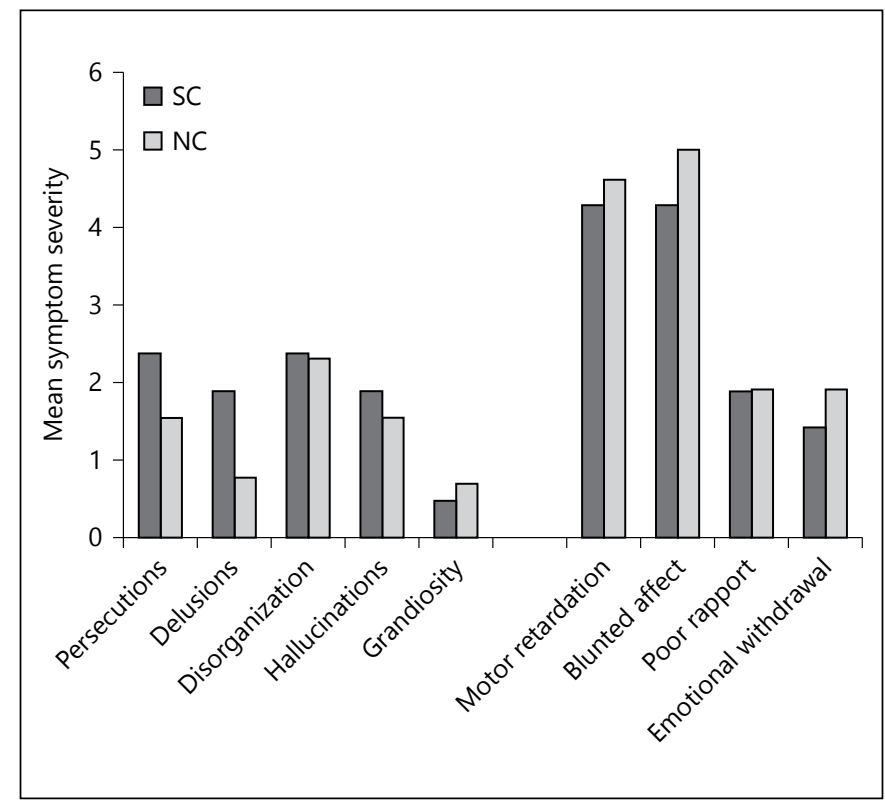

Fig. 4. Comparisons of the mean severity of symptoms between drug types. $1=$ Absent, $2=\operatorname{minimal}, 3=$ mild, $4=$ moderate, $5=$ moderately severe, $6=$ severe, $7=$ extreme.

symptoms was significantly greater during drug use than before or after drug use. There was no difference between men and women in the severity or duration of the effects induced by the 2 drugs in either the psychotic or non-psychotic patient population.

\section{Influence of Psychotic Illness}

Prevalence

For the statistical analyses and multivariate regression analysis, the following diagnoses were considered part of the schizophrenic symptom complex: paranoid schizophrenia, schizoaffective disorder, schizophreniform disorder, major depressive disorder with psychotic features and disorganized schizophrenia (Table 1). Chisquare tests comparing the prevalence rates of acute psychotic reactions between psychotic and non-psychotic patients showed that psychotic illness was not significantly associated with acute psychosis during drug use.

\section{Severity}

We assessed the impact of psychotic illness on symptom severity and found that acute motor retardation was significantly more severe among non-psychotic patients than among psychotic patients $(U=134.00, p=0.005)$, whereas acute delusions of persecution were more severe among psychotic patients than among non-psychotic patients $(\mathrm{U}=176.00, p=0.019)$.
Influence of Drug Use Frequency

To determine whether drug use frequency affected the frequency of psychotic reactions, we assessed the correlations between the frequency of each drug use and the prevalence of each symptom included in the study. Bonferroni correction was applied to account for multiple correlations, and the significance level was set at 0.006 . Frequent drug use was associated with delusions of persecution $(r=0.37, p=0.010)$, disorganization $(r=0.73$, $p>0.001)$, blunted affect $(r=0.75, p<0.001)$, and motor retardation $(r=0.495, p<0.001)$.

Regarding the relationship between drug use frequency and symptom severity, more frequent drug use was significantly associated with more severe acute delusions $(F[1,19]=30.43, p<0.001)$, grandiosity $(F[1,19]=12.43$, $p=0.002)$, and disorganization $(F[1,19]=31.90, p<$ $0.001)$.

\section{Discussion}

This prospective study was one of the first to assess the prevalence of SC use, the profiles of SC users, and the psychosis-inducing potential of SCs and to compare these results to those of NC and $\mathrm{NC}$ users in a naturalistic psychiatric population. Given the scarcity of studies on the symptomatology of SC-induced psychosis and the effects of SCs and NC on psychosis, we examined the symptoms experienced by SC and NC users. Previous studies have shown that the rate of $\mathrm{NC}$ use is higher among psychotic patients than among the general population [31] and that psychosis-prone individuals seem to experience greater numbers of drug-related psychotic reactions than those who are not prone to psychosis $[11,18,20,44-46]$. Thus, we were particularly interested in assessing the relative risks of SC- and NC-related psychosis in psychotic and non-psychotic patients.

The lifetime prevalence of SC use in the total sample was high (7.2\%) and was higher among psychotic patients $(10.6 \%)$ than among non-psychotic patients. However, it is difficult to determine whether these findings are applicable to the general population because few studies on the prevalence of SC use among psychotic and non-psychotic patients have been conducted, and those that have been performed have investigated a limited number of subpopulations in a limited number of countries.

The consumer profiles established in this study were similar to those hypothesized, as we determined that smoking, young age, and frequency of $\mathrm{NC}$ consumption were risk factors for SC use. Based on our reviews of SC 
case reports, we hypothesized that most SC users would be young male users of other drugs, including NC. Patients' gender predicted SC use with only marginal significance, and male patients tended to be more likely than female patients to use SCs. NC was the only drug that significantly predicted $\mathrm{SC}$ use, indicating that substances, with the exception of NC and tobacco, do not seem to increase the risk of SC use. This finding is in line with the results of previous studies that report a strong association between NC use and SC use $[47,48]$. Moreover, the results of this study showed that the majority of SC users in our population were previous NC users who tried SCs. However, these results should be interpreted with caution because of the small sample size and should be confirmed in a larger, systematic prospective study.

Of the SC and NC users with psychosis, 76.2 and $80.8 \%$, respectively, reported experiencing at least one of the 9 psychotic symptoms assessed during SC and NC exposure. Both SC and NC users experienced negative symptoms more frequently than they experienced positive symptoms. Although we hypothesized that SC-related psychosis would be more prevalent and more severe than NC-related psychosis among patients enrolled in the study, this hypothesis was not supported by the results. Positive symptoms were slightly more frequent among SC users than among NC users; however, overall, we noted no differences in symptoms between the 2 groups.

The lack of significant findings in this study may have been due to its small sample size, which should be addressed by future studies. Interestingly, negative psychotic symptoms tended to be slightly more common among NC users than among SC users. Somewhat consistent with this finding, we found that $\mathrm{NC}$ users experienced significantly more severe negative symptoms than SC users. Although we hypothesized that psychotic patients would be more susceptible to acute psychotic reactions than non-psychotic patients, this hypothesis was not supported for use of either drug. However, the small sample size may have affected the statistical significance of this relationship.

Two SC users recalled experiencing positive symptoms that first occurred during SC use and persisted after the cessation of drug use. Both patients had a history of heavy NC use and a family history of psychotic illness. One of the patients had been diagnosed with paranoid schizophrenia before SC use, while the other patient had no history of mental illness. These findings should be interpreted with caution, as although the patients' symptoms occurred for the first time during SC exposure, both patients had a history of heavy cannabis use and a per- sonal and/or familial history of psychotic illness. Therefore, these cases are not clear-cut examples of SC-induced psychosis and should not be used to draw conclusions regarding a causal relationship between SC use and psychotic symptoms.

Due to the difficulty of identifying truly separate groups of drug users (i.e., groups comprising patients who used only SCs or only NC), previous studies have faced problems similar to those encountered in this study when interpreting their results. In all the case reports referred to in this text, patients with new-onset SC-related psychosis had previously used NC or other drugs. Fattore and Fratta [13] addressed the possibility that SC use may have triggered the onset of undetected prodromal psychotic symptoms in some patients. Thus, it is possible that the use of other drugs induces the development of a pre-symptomatic condition that can also be triggered by SC use. Accordingly, in the patients assessed in this study, SC use may have been merely the "final straw" in a series of events leading to the manifestation of a condition with multiple unrelated causes. This hypothesis is supported by the finding that SCs have a greater affinity for CB1 receptors than tetrahydrocannabinol, the main psychoactive compound in NC.

Analyses regarding the relationship between drug exposure and psychotic symptoms are also complicated by the fact that many years can pass between a patient's first drug exposure and the emergence of psychotic symptoms, as shown in a study by McGrath et al. $[49,50]$ in which psychosis first manifested in patients at an average of 7-8 years after their first use of cannabis.

Similar to the cases of SC-induced psychosis discussed in this study, the cases of NC-induced psychosis were precipitated by heavy cannabis use. Furthermore, the likelihood of experiencing delusions of persecution, disorganization, blunted affect, and motor symptoms increased as the frequency of prior NC or SC consumption increased. Similarly, frequent drug use was associated with more severe positive symptoms among users of both drugs. These findings are consistent with those of previous studies on $\mathrm{NC}$, which found that heavier cannabis use was associated with a higher risk of psychosis $[22,51,52]$. Due to its greater potency, $\mathrm{SC}$ use is believed to be a significant risk factor for the development of more severe psychotic symptoms. However, larger systematic controlled studies must be performed to confirm this hypothesis.

The repeated measures analyses revealed that in both NC and SC users, patients' symptoms did not significantly worsen between the pre- and post-drug use time points. Rather, their symptoms were significantly more severe 
during intoxication than before or after intoxication, indicating that SC and NC use causes acute but not prolonged exacerbations of psychotic symptoms. However, as these patients experienced symptoms before using SCs or NC, it is likely that they were receiving antipsychotic medications while using SCs or NC or had received similar medications during that time frame. These medications may have prevented patients' symptoms from worsening after cessation of drug use.

\section{Limitations}

The findings of this study should be interpreted with caution given the several methodological limitations; the most important limitation was the size of the SC and NC user samples, which were too small to draw definitive conclusions. Moreover, the SC and NC user groups did not include control subgroups comprising non-psychiatric $\mathrm{NC} / \mathrm{SC}$ users.

Additionally, we did not compare the results of the above groups with those of a (larger) group of non-psychiatric patients. Instead, we compared the observed prevalence rates of SC use with the estimated prevalence rates of SC use in previous studies. The studies in question estimated that the prevalence of SC use was $1 \%$ in the general population, $2-10 \%$ among students and $10 \%$ among groups with high rates of drug use (e.g., nightclubbers) $[45,47,48,53,54]$. Compared with these estimated rates, the prevalence of SC use in the present sample seems very high. However, the groups examined herein were at high risk of using SCs. Thus, it is not surprising that our observed percentage of patients with a history of SC use was higher than that in the general population. Several previous studies have reported that the prevalence of cannabis and SC use is higher among psychotic patients than among non-psychotic patients $[45,47,48,53,54]$, and these results were supported by the results of this study.

As mentioned above, interpreting the relationship between drug use and psychosis in multidrug users is difficult. Considering the fact that SCs, such as NC, may exhibit psychosis-inducing effects only years after they are first consumed, it is virtually impossible to tie specific drug-related effects to specific drugs in persons with a history of multidrug use, even when the individual used each drug separately. Another inherent limitation of studies utilizing self-reported data is the subjective nature of the histories reported by the patients. Some of the patients enrolled in this study described drug use-related experiences dating back several years. We attempted to maximize the accuracy of the patients' retrieved memories by prompting them during their interviews; however, it is possible that patients experiencing delusions are more prone than patients without delusions to attribute their symptoms to an external cause. Previous studies have shown that externalizing bias can affect the results of studies involving psychotic patients [55].

\section{Conclusions}

This study presented additional evidence of the risks of both SC and NC use. The prevalence of SC use among psychotic and non-psychotic patients was significant, and several psychotic symptoms seemed to appear in the majority of SC users. Our findings indicate that both NC and SCs can induce persistent psychosis, as patients in both groups reported that they experienced symptoms for the first time during intoxication and that the symptoms persisted after drug use cessation. Unfortunately, it is not possible to draw any conclusions regarding causal relationships between the different variables, as all SC consumers in this study had previously used NC.

With regard to the relationship between SC use and psychotic symptoms, we also found that specific symptom clusters were associated with each drug type. The observed differences in the symptomatology of SC- and NC-related psychosis seem to be worth exploring in future investigations, especially those aiming to determine whether the different psychotic symptoms associated with SC and NC use warrant different (clinical) treatments.

In addition to enrolling larger samples, future studies should assess groups comprising only SC and NC users by applying the abovementioned questionnaires and should compare these results to those of carefully matched patients without psychiatric illnesses. Given the high frequency of concomitant NC and SC use, laboratory studies may be required to truly control for the effects of prior NC use. Researchers can also control for prior NC use by matching study groups (NC/SC vs. only NC) according to their frequencies of previous NC use. As no information about the specific temporal trajectories of drug use in our patients was collected, matching subjects according to their frequencies of prior NC use was not feasible in this study.

Ultimately, long-term follow-up studies utilizing more direct data collection methods are needed to shed light on the late effects of SCs.

In conclusion, the present study examined an understudied research topic, and the results indicated that additional research is needed to better understand the role of 
SCs in psychosis. Notably, the prevalence of SC use was very high in psychiatric patients and should be monitored further in subsequent studies. Finally, teenagers and young adults seem to know little about the adverse effects of NPS. Therefore, it is important to educate these groups about the dangers associated with psychoactive substance use.

\section{Acknowledgment}

None.

\section{Ethics Approval, Registration, and Consent to Participate}

Informed written consent was obtained from each participant who agreed to participate in the study at the time of recruitment. The participants were aware that they could withdraw from the study at any time and were assured that their data would remain confidential. The study was approved by the Ethics Commission of the Medical Faculty of the Carl von Ossietzky University of Oldenburg $(\mathrm{CvO})$.

\section{Consent for Publication}

Not applicable.

\section{Availability of Data and Materials}

The data supporting our findings are available from the corresponding author upon request.

\section{Disclosure Statement}

The authors declare that they have no competing interests regarding the publication of this manuscript.

\section{Funding Sources}

No special funding was provided for this research.

\section{Author Contributions}

S.W. performed the data collection and statistical analyses and wrote a draft of the manuscript. H.H.O.M., A.P., and S.W. conceptualized the paper and wrote the final draft of the manuscript. C.M.T. and H.H.O.M. provided expertise regarding the methodological and statistical procedures used in the study. C.L., C.C., A.P.L., S.M., H.H.O.M., and P.S. provided expertise regarding the relationship between NC use and psychosis. A.P. and H.H.O.M. critically revised the manuscript. All authors contributed to the writing of the manuscript and have read and approved of the final manuscript.

\section{References}

1 Khaled SM, Hughes E, Bressington D, Zolezzi M, Radwan A, Badnapurkar A, Gray R: The prevalence of novel psychoactive substances (NPS) use in non-clinical populations: a systematic review protocol. Syst Rev 2016;5:195.

2 Betzler F, Heinz A, Köhler S: [Synthetic drugs - an overview of important and newly emerging substances]. Fortschr Neurol Psychiatr 2016;84:690-698.

3 Winstock A, Lynskey M, Borschmann R, Waldron J: Risk of emergency medical treatment following consumption of cannabis or synthetic cannabinoids in a large global sample. J Psychopharmacol 2015;29:698-703.

4 Le Boisselier R, Alexandre J, Lelong-Boulouard V, Debruyne D: Focus on cannabinoids and synthetic cannabinoids. Clin Pharmacol Ther 2016;101:220-229.

5 Schifano F, Orsolini L, Papanti D, Corkery J: NPS: medical consequences associated with their intake. Curr Top Behav Neurosci 2017; $32: 351-380$

6 De Luca MA, Castelli MP, Loi B, Porcu A, Martorelli M, Miliano C, Kellett K, Davidson C, Stair JL, Schifano F, Di Chiara G: Native CB1 receptor affinity, intrinsic activity and accumbens shell dopamine stimulant properties of third generation SPICE/K2 cannabinoids: BB-22, 5F-PB-22, 5F-AKB-48 and STS-135. Neuropharmacology 2016; 105: 630-638.

7 Karila L, Benyamina A, Blecha L, Cottencin $\mathrm{O}$, Billieux J: The synthetic cannabinoids phenomenon. Curr Pharm Des 2016;22:64206425.

8 Müller H, Huttner HB, Köhrmann M, Wielopolski JE, Kornhuber J, Sperling W: Panic attack after spice abuse in a patient with ADHD. Pharmacopsychiatry 2010;43:152-153.

9 Müller H, Sperling W, Köhrmann M, Huttner HB, Kornhuber J, Maler JM: The synthetic cannabinoid Spice as a trigger for an acute exacerbation of cannabis induced recurrent psychotic episodes. Schizophr Res 2010;118: 309-310.

10 Tai S, Fantegrossi WE: Pharmacological and toxicological effects of synthetic cannabinoids and their metabolites. Curr Top Behav Neurosci 2017;32:249-262.

11 Müller HH, Kornhuber J, Sperling W: The behavioral profile of spice and synthetic cannabinoids in humans. Brain Res Bull 2016; 126(pt 1):3-7.
12 Adams AJ, Banister SD, Irizarry L, Trecki J, Schwartz M, Gerona R: "Zombie" outbreak caused by the synthetic cannabinoid AMBFUBINACA in New York. N Engl J Med 2017; 376:235-242.

13 Fattore L, Fratta W: Beyond THC: the new generation of cannabinoid designer drugs. Front Behav Neurosci 2011;5:60.

14 Schaefer N, Kettner M, Laschke MW, Schlote J, Ewald AH, Menger MD, Maurer $\mathrm{HH}$, Schmidt PH: Distribution of synthetic cannabinoids JWH-210, RCS-4 and $\Delta$ 9-tetrahydrocannabinol after intravenous administration to pigs. Curr Neuropharmacol 2017;15:713-723.

15 Springer YP, Gerona R, Scheunemann E, Shafer SL, Lin T, Banister SD, Cooper MP, Castrodale LJ, Levy M, Butler JC, McLaughlin JB: Increase in adverse reactions associated with use of synthetic cannabinoids - Anchorage, Alaska, 2015-2016. MMWR Morb Mortal Wkly Rep 2016;65:1108-1111.

16 Joseph AM, Manseau MW, Lalane M, Rajparia A, Lewis CF: Characteristics associated with synthetic cannabinoid use among patients treated in a public psychiatric emergency setting. Am J Drug Alcohol Abuse 2016;43: 117-122. 
17 Haden M, Archer JR, Dargan PI, Wood DM: MDMB-CHMICA: availability, patterns of use, and toxicity associated with this novel psychoactive substance. Subst Use Misuse 2017;52:223-232.

18 Murray RM, Quigley H, Quattrone D, En glund A, Di Forti M: Traditional marijuana, high-potency cannabis and synthetic cannabinoids: increasing risk for psychosis. World Psychiatry 2016;15:195-204.

19 Murray RM, Di Forti M: Cannabis and psychosis: what degree of proof do we require? Biol Psychiatry 2016;79:514-515.

20 Fattore L: Synthetic cannabinoids-further evidence supporting the relationship between cannabinoids and psychosis. Biol Psychiatry 2016;79:539-548.

21 Van der Veer N, Friday J: Persistent psychosis following the use of Spice. Schizophr Res 2011;130:285-286.

22 D'Souza DC, Radhakrishnan R, Sherif M Cortes-Briones J, Cahill J, Gupta S, Skosnik PD, Ranganathan M: Cannabinoids and psychosis. Curr Pharm Des 2016;22:6380-6391.

23 Gray R, Bressington D, Hughes E, Ivanecka A A systematic review of the effects of novel psychoactive substances 'legal highs' on people with severe mental illness. J Psychiatr Ment Health Nurs 2016;23:267-281.

24 Sherif M, Radhakrishnan R, D'Souza DC, Ranganathan M: Human laboratory studies on cannabinoids and psychosis. Biol Psychiatry 2016;79:526-538.

25 Albertson TE, Chenoweth JA, Colby DK, Sutter ME: The changing drug culture: Emerging drugs of abuse and legal highs. FP Essent 2016;441:18-24.

26 Albertson TE, Chenoweth JA, Colby DK, Sutter ME: The changing drug culture: medical and recreational marijuana. FP Essent 2016; 441:11-17.

27 Ernst L, Schiebel HM, Theuring C, Lindigkeit $\mathrm{R}$, Beuerle T: Identification and characterization of JWH-122 used as new ingredient in "Spice-like" herbal incenses. Forensic Sci Int 2011;208:e31-e35.

28 Johnson LA, Johnson RL, Alfonzo C: Spice: a legal marijuana equivalent. Mil Med 2011; 176:718-720.

29 Forrester MB, Kleinschmidt K, Schwarz E, Young A: Synthetic cannabinoid and marijuana exposures reported to poison centers. Hum Exp Toxicol 2012;31:1006-1011.

30 van Amsterdam J, Brunt T, van den Brink W: The adverse health effects of synthetic cannabinoids with emphasis on psychosis-like effects. J Psychopharmacol 2015;29:254-263.
31 Green B, Young R, Kavanagh D: Cannabis use and misuse prevalence among people with psychosis. Br J Psychiatry 2005;187:306-313.

32 Henquet C, Murray R, Linszen D, van Os J: The environment and schizophrenia: the role of cannabis use. Schizophr Bull 2005;31:608612.

33 Henquet C, Krabbendam L, Spauwen J, Kaplan C, Lieb R, Wittchen HU, van Os J: Prospective cohort study of cannabis use, predisposition for psychosis, and psychotic symptoms in young people. BMJ 2005;330:11.

34 Andrade C: Cannabis and neuropsychiatry, 2: the longitudinal risk of psychosis as an adverse outcome. J Clin Psychiatry 2016; 77:e739-e742.

35 Andrade C: Cannabis and neuropsychiatry, 1: benefits and risks. J Clin Psychiatry 2016; 77:e551-e554.

36 Wilkinson ST, Radhakrishnan R, D'Souza DC: Impact of cannabis use on the development of psychotic disorders. Curr Addict Rep 2014;1:115-128.

37 van Winkel R, Kuepper R: Epidemiological, neurobiological, and genetic clues to the mechanisms linking cannabis use to risk for nonaffective psychosis. Annu Rev Clin Psychol 2014;10:767-791

38 Papanti D, Schifano F, Botteon G, Bertossi F, Mannix J, Vidoni D, Impagnatiello M, Pascolo-Fabrici E, Bonavigo T: "Spiceophrenia": a systematic overview of "spice"-related psychopathological issues and a case report. Hum Psychopharmacol 2013;28:379-389.

39 Renard J, Krebs MO, Le Pen G, Jay TM: Longterm consequences of adolescent cannabinoid exposure in adult psychopathology. Front Neurosci 2014;8:361.

40 Donoghue K, Doody GA, Murray RM, Jones PB, Morgan C, Dazzan P, Hart J, Mazzoncini $\mathrm{R}$, Maccabe JH: Cannabis use, gender and age of onset of schizophrenia: data from the ÆSOP study. Psychiatry Res 2014;215:528532.

41 Every-Palmer S: Synthetic cannabinoid JWH018 and psychosis: an explorative study. Drug Alcohol Depend 2011;117:152-157.

42 Evins AE, Green AI, Kane JM, Murray RM: The effect of marijuana use on the risk for schizophrenia. J Clin Psychiatry 2012;73: 1463-1468.

43 Kay SR, Fiszbein A, Opler LA: The positive and negative syndrome scale (PANSS) for schizophrenia. Schizophr Bull 1987;13:261276
44 Haro G, Ripoll C, Ibáñez M, Orengo T, Liaño VM, Meneu E, Hernández F, Traver F: Could spice drugs induce psychosis with abnormal movements similar to catatonia? Psychiatry 2014;77:206-208

45 Loeffler G, Delaney E, Hann M: International trends in spice use: prevalence, motivation for use, relationship to other substances, and perception of use and safety for synthetic cannabinoids. Brain Res Bull 2016;126:8-28.

46 Brakoulias V: Products containing synthetic cannabinoids and psychosis. Aust N Z J Psychiatry 2012;46:281-282.

47 Bretteville-Jensen AL, Tuv SS, Bilgrei OR, Fjeld B, Bachs L: Synthetic cannabinoids and cathinones: prevalence and markets. Forensic Sci Rev 2013;25:7-26.

48 Kikura-Hanajiri R, Kawamura NU, Goda Y: Changes in the prevalence of new psychoactive substances before and after the introduction of the generic scheduling of synthetic cannabinoids in Japan. Drug Test Anal 2014; 6:832-839.

49 McGrath JJ, Mortensen PB, Visscher PM Wray NR: Where GWAS and epidemiology meet: opportunities for the simultaneous study of genetic and environmental risk factors in schizophrenia. Schizophr Bull 2013;39: 955-959.

50 McGrath J, Meyer-Lindenberg A: Is it time schizophrenia research left the museum? Clin Schizophr Relat Psychoses 2013;6:170-171.

51 Fergusson DM, Horwood LJ, Ridder EM: Tests of causal linkages between cannabis use and psychotic symptoms. Addiction 2005; 100:354-366.

52 D'Souza DC: Cannabinoids and psychosis. Int Rev Neurobiol 2007;78:289-326.

53 Tuv SS, Krabseth H, Karinen R, Olsen KM Øiestad EL, Vindenes V: Prevalence of synthetic cannabinoids in blood samples from Norwegian drivers suspected of impaired driving during a seven weeks period. Accid Anal Prev 2014;62:26-31.

54 Heltsley R, Shelby MK, Crouch DJ, Black DL, Robert TA, Marshall L, Bender CL, DePriest AZ, Colello MA: Prevalence of synthetic cannabinoids in U.S. athletes: initial findings. J Anal Toxicol 2012;36:588-593.

55 Van Dael F, Versmissen D, Janssen I, MyinGermeys I, van Os J, Krabbendam L: Data gathering: biased in psychosis? Schizophr Bull 2006;32:341-351

56 van Os J, Bak M, Hanssen M, Bijl RV, de Graaf $\mathrm{R}$, Verdoux H: Cannabis use and psychosis: longitudinal population-based study. Am J Epidemiol 2002;156:319-327. 